\title{
ALIGNTTS: EFFICIENT FEED-FORWARD TEXT-TO-SPEECH SYSTEM WITHOUT EXPLICIT ALIGNMENT
}

\author{
Zhen Zeng, Jianzong Wang*, Ning Cheng, Tian Xia, Jing Xiao
}

Ping An Technology (Shenzhen) Co., Ltd.

\begin{abstract}
Targeting at both high efficiency and performance, we propose AlignTTS to predict the mel-spectrum in parallel. AlignTTS is based on a Feed-Forward Transformer which generates mel-spectrum from a sequence of characters, and the duration of each character is determined by a duration predictor. Instead of adopting the attention mechanism in Transformer TTS to align text to mel-spectrum, the alignment loss is presented to consider all possible alignments in training by use of dynamic programming. Experiments on the LJSpeech dataset show that our model achieves not only state-of-the-art performance which outperforms Transformer TTS by 0.03 in mean option score (MOS), but also a high efficiency which is more than 50 times faster than real-time.
\end{abstract}

Index Terms - text-to-speech, speech synthesis, feedforward transformer, Baum-Welch algorithm

\section{INTRODUCTION}

Recent advances in text-to-speech (TTS) are driven by the success of autoregressive neural network, such as Char2wav [1], VoiceLoop [2], Tacotron [3], Tacotron 2 [4], Deep Voice 1 [5],Deep Voice 2 [6], Deep Voice 3 [7], Clarinet [8] and Transformer TTS [9]. These models employ a sequence-tosequence (seq2seq) framework with attention mechanism, where the encoder converts the input text into a contextsensitive features and the decoder generates the mel-spectrum based on the context-sensitive features. In addition, a neural vocoder, such as WaveNet [10], WaveRNN [11] and WaveGlow [12], is usually used to synthesize waveform from the mel-spectrum. Although the autoregressive-based TTS system has achieved satisfactory synthesis results, low inference efficiency of autoregressive neural network still limits its application in a real-time dialogue system.

Most recently, many non-autoregressive architectures for TTS are proposed to increase the speed of synthesis speech, such as ParaNet [13] and FastSpeech [14]. Combining with parallel neural vocoder, these models can achieve real-time speech synthesis by predicting mel-spectrum in parallel. However, since it is difficult for these models to learn the alignment between text and mel-spectrum, a pre-trained

\footnotetext{
*Corresponding author: Jianzong Wang, jzwang@188.com
}

auto-regressive model is required to guide their training, which limits them to achieve better performance. Therefore, in order to improve the quality of synthesis speech, it is necessary for non-autoregressive TTS systems to design more appropriate method to directly learn the alignment.

Reviewing the traditional statistical parametric speech synthesis system based on the Hidden Markov Models (HMMs) [15, 16], the Baum-Welch algorithm [17] is used to estimate the parameters of HMMs if the alignment between states and observations is unknown. In detail, the Baum-Welch algorithm considers all possible alignments and sums the estimated value from each alignment as the final estimated value, where each observation is assigned to every state in proportion to probability of that generating the observation. Inspired by this idea, we present a novel method to improve the alignment for the non-autoregressive TTS system.

In this paper, we propose AlignTTS to generate the melspectrum in parallel, whose training does not require guidance from other autoregressive TTS systems. AlignTTS consists of a Feed-Forward Transformer, a duration predictor and a mix density network. The Feed-Forward Transformer is a feedforward network to transform text to mel-spectrum, where the duration of each character predicted by the duration predictor is required to regulate the alignment in inference. Experiments on the LJSpeech dataset show that AlignTTS achieves state-of-art performance which outperforms Transformer TTS by a gap of 0.03 in MOS. Meanwhile, it takes only 0.18 seconds to synthesize approximately 10 seconds of speech in our model, which is more than 50 times faster than real-time. And the main contributions of our works as follows:

- Due to the feed-forward network structure, AlignTTS can generate the mel-spectrum in parallel. Combining with WaveGlow Vocoder, the speech synthesis speed is more than 50 times faster than real-time;

- The alignment loss is proposed to guide AlignTTS to learn the alignment between the text and mel-spectrum. Specifically, the learned alignment is more precise in aligning text and mel-spectrum than the attention alignment from Transformer TTS [9], so that the more accurate conversion from text to mel-spectrum is learned in AlignTTS. 


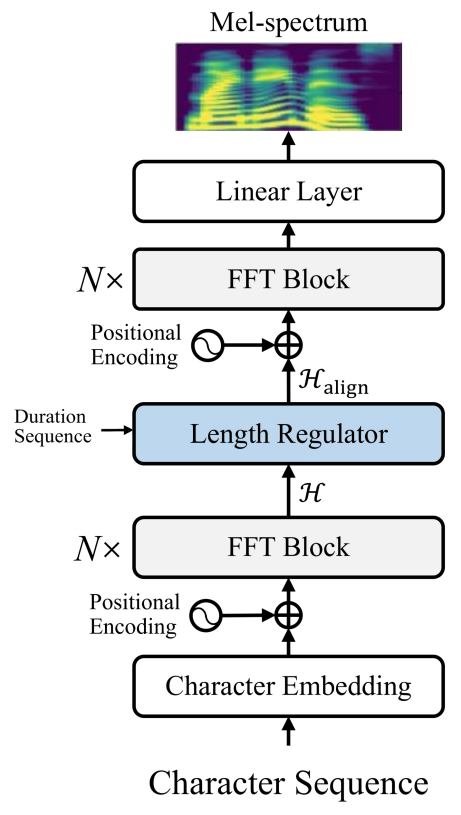

(a) Feed-Forward Transformer

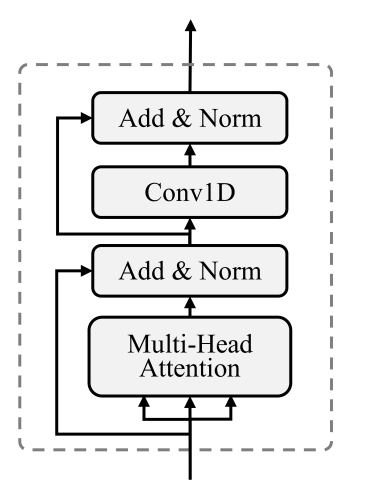

(b) FFT Block

Fig. 1. (a) Feed-Forward Transformer for text to melspectrum transformation. (b) FFT Block, composed of selfattention and 2-layer 1D conventional network.

\section{ARCHITECTURE OF ALIGNTTS}

In order to predict the mel-spectrum in parallel, we propose AlignTTS, a feed-forward network, which contains three modules including the Feed-Forward Transformer module, the duration predictor and the mix density network. Each module is described in detail in this section.

\subsection{Feed-Forward Transformer}

The Feed-Forward Transformer (FFT) module stacks a character embedding, multiple FFT blocks, a length regulator and a linear layer for text to mel-spectrum transformation, as shown in Figure 1(a). Multiple FFT blocks are divided into two parts by the length regulator. Each FFT block is composed of a self-attention from Transformer [18] and 2layer 1D convolutional network, where residual connections, layer normalization and dropout are used, as shown in Figure 1(b). In addition, the length regulator is used to regulate the alignment between text and mel-spectrum according to the given duration sequence, which is generated by the duration predictor in inference. Since the adjustment method of the length regulator is the same as description in FastSpeech [14], the voice speed and the breaks between words can also be controlled in our model.

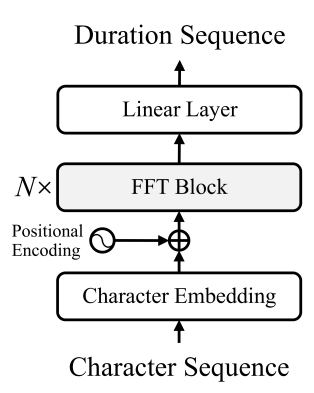

(a) Duration Predictor

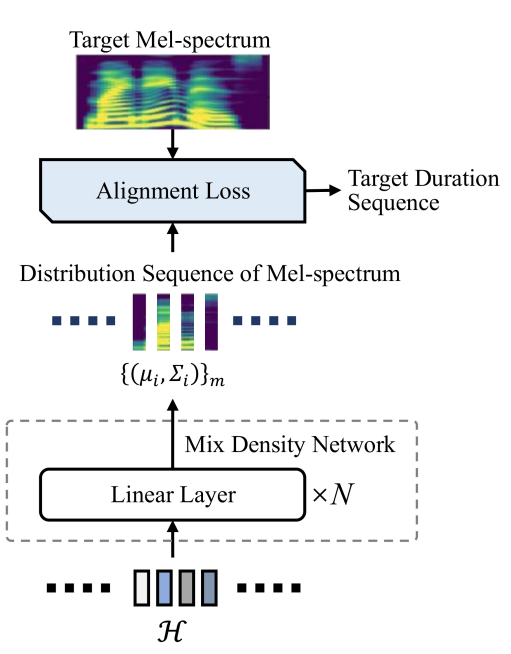

(b) Mix Density Network

Fig. 2. (a) The duration predictor for predicting the duration sequence in inference. (b) The Mix Density Network for learning the alignment between text and mel-spectrum.

\subsection{Duration Predictor}

Different from conventinal speech synthesis systems employing the attention mechanism, AlignTTS uses the duration predictor to predict the alignment between text and melspectrum, which can be calculated in parallel. The duration predictor consists of a character embedding layer, multiple stacked FFT blocks and a linear network to output a scalar, as shown in Fig. 2(a). According to the input character sequence, the duration predictor outputs a duration sequence, where each element of the duration sequence represents the pronunciation duration of each character. In inference, the output duration sequence is used in the length regulator to control the alignment between text and mel-spectrum.

\subsection{Mix Density Network}

Since the training of Feed-Forward Transformer and the duration predictor requires the correct alignment between the text and mel-spectrum of training dataset, we design the mix density network [19, 20] to learn the alignment. As shown in Fig. 2(b), the mix density network is composed of multiple stacked linear layers, each followed by the layer normalization, ReLU activation and the dropout layer but the last. The last linear layer outputs the mean and variance vector of multi-dimensional gaussian distributions, which represents the mel-spectrum distribution of each character. Note that the mix density network is stacked on top of the FFT blocks on the character side in Feed-Forward Transformer, and its input is the same with the length regulator. In addition, the mix density network is designed to learn the alignment of training dataset, hence it is only used in the model training stage and can be removed in inference. 


\section{TRAINING AND INFERENCE}

\subsection{Alignment Loss}

Inspired by the Baum-Walch algorithm [16, 17], we propose the alignment loss to train the mix density network and learn the correct alignment between text and mel-spectrum. Let $\boldsymbol{y}=\left\{y_{1}, y_{2}, \cdots, y_{n}\right\}$ denote the mel-spectrum sequence, where $n$ is the frame number of the mel-spectrum. And let $\boldsymbol{z}=\left\{z_{1}, z_{2}, \cdots, z_{m}\right\}$ denote the multi-dimensional gaussian distribution sequence output from the mix density network, where $z_{i}=\left(\mu_{i}, \Sigma_{i}\right)$ represents mean vector and covariance matrix, and $m$ is equal to the length of input character sequence. We consider the distribution of each dimensional in the mel-spectrum is independent, so the covariance matrix $\Sigma_{i}$ is a diagonal matrix. The probability of the $i$-th frame mel-spectrum with respect to the $j$-th distribution of the multi-dimensional gaussian distribution sequence can be calculated by the multi-dimensional gaussian function:

$$
p\left(y_{i} \mid z_{j}\right)=\mathcal{N}\left(y_{i} \mid \mu_{j}, \Sigma_{j}\right)
$$

Let $\ell$ denote the alignment, and $j=\operatorname{Align}(i, \ell)$ denote that the $i$-th frame mel-spectrum is aligned to the $j$-th element of the distribution sequence. The objective function can be considered as the condition probability of the mel-spectrum sequence $\boldsymbol{y}$ with respect to the distribution sequence $\boldsymbol{z}$ :

$$
p(\boldsymbol{y}, \ell \mid \boldsymbol{z})=\prod_{i=0}^{n} p\left(y_{i} \mid z_{\operatorname{Align}(i, \ell)}\right)
$$

However, due to the unknown of the alignment, we cannot directly calculate the above equation. Fortunately, the BaumWelch algorithm provides a great idea to solve this problem, which considers all possible alignment and sums their probability. Therefore, the objective function is designed as

$$
p(\boldsymbol{y} \mid \boldsymbol{z})=\sum_{\ell} \prod_{i=0}^{n} p\left(y_{i} \mid z_{\operatorname{Align}(i, \ell)}\right)
$$

Finally, the alignment loss is designed as

$$
\mathcal{L}_{\text {align }}(\boldsymbol{z}, \boldsymbol{y})=-\log p(\boldsymbol{y} \mid \boldsymbol{z})
$$

When the alignment loss converges, the correct alignment can be extracted using the Viterbi algorithm [16].

\subsection{Training}

\subsubsection{Forward Algorithm for Alignment Loss}

Similar to the forward-backward algorithm for HMMs, we apply a dynamic programming algorithm to efficiently calculate the loss. Define the forward variables as

$$
\alpha_{t, s}=p\left(\boldsymbol{y}_{1: t} \mid \boldsymbol{z}_{1: s}\right)
$$

where $\boldsymbol{y}_{1: t}=\left\{y_{1}, y_{2}, \cdots, y_{t}\right\}, 0<t \leq n$ and $\boldsymbol{z}_{1: s}=$ $\left\{z_{1}, z_{2}, \cdots, z_{s}\right\}, 0<s \leq m$. The following recursion is established

$$
\alpha_{1,1}=p\left(y_{1} \mid z_{1}\right), \quad \alpha_{1, s}=0 \quad \forall 1<s \leq m
$$

and $\forall 1<t \leq n, 1 \leq s \leq m$,

$$
\alpha_{t, s}=\left(\alpha_{t-1, s}+\alpha_{t-1, s-1}\right) \cdot p\left(y_{t} \mid z_{s}\right)
$$

Then the alignment loss can be calculated by the above recursive formula:

$$
\mathcal{L}_{\text {align }}(\boldsymbol{z}, \boldsymbol{y})=-\log p\left(\boldsymbol{y}_{1: n} ; \boldsymbol{z}_{1: m}\right)=-\log \alpha_{n, m}
$$

Note that the alignment loss is differentiable with respect to the distribution sequence $z$ since its just sums, products and exponents of them. Therefore, the mix density network can be trained using the gradient descent algorithm applied by Tensorflow or Pytorch.

\subsubsection{Multi-phases Training}

Since the training of Feed-Forward Transformer and the duration predictor requires the correct alignments which are obtained from the trained mix density network, it is difficult to jointly train the entire network at once in practice. Therefore, we adopt multi-phases training for AlignTTS.

- Firstly, we train the mix density network with the first FFT blocks of the Feed-Forward Transformer using the alignment loss.

- Secondly, we extract the alignment and convert it into the duration sequence. Fixing the parameters of the first FFT blocks, the rest network of Feed-Forward Transformer is trained using the mean square error (MSE) loss between the predicted and target mel-spectrum.

- Then, the Feed-Forward Transformer and the mix density network are trained together to fine-tune parameters, where the duration sequence is calculated in each training step.

- Finally, we use the final mix density network to calculate the character durations and train the duration predictor using the MSE loss in logarithmic domain [14].

\subsection{Inference}

In inference, input characters are convert to $N$-dimensional character embedding sequence which are passed through the first FFT blocks of Feed-Forward Transformer to output the hidden feature sequence. At the same time, the duration predictor generates the duration sequence, which is used by the length regulator to expand the hidden feature sequence. The rest network of Feed-Forward Transformer predicts the melspectrum based on the expanded hidden feature sequence. Finally, a parallel neural vocoder, such as WaveGlow, is used to generate waveform according to the predicted mel-spectrum. 


\section{EXPERIMENTS}

\subsection{Datasets}

We train AlignTTS on LJSpeech dataset [21], which is randomly divided into 2 sets: 12600 samples for training, and 500 samples for testing. The computation of the melspectrum is the same with the description in WaveGlow [12]. The texts are normalized and inserted with the beginning character (a space) and the end character (a period), e.g. "There are 16 apples" is converted to "there are sixteen apples.".

\subsection{Model Configuration}

The Feed-Forward Transformer contains 6 FFT blocks on both the character side and the mel-spectrum side, and the duration predictor includes 2 FFT blocks. The dimension of each network in the Feed-Forward Transformer is all set to 768 and the dimension in the duration predictor is all set to 128. The number of attention head is set to 2 and the kernel size of 1D convolution is set to 3 in all FFT block. The hidden size of the linear layer in the mix network is set to 256 and the dimension of the output is 160 ( 80 dimensions for the mean and 80 dimensions for variance of the gaussian distribution).

We train our model on 2 NVIDIA V100 GPUs, with batch size of 16 samples on each GPU, and use the Adam optimizer with $\beta_{1}=0.9, \beta_{2}=0.98, \varepsilon=10^{-9}$. we adopt the same learning rate schedule in [18] with $40 \mathrm{~K}$ training steps in the first two training stages, and a fixed learning rate of $10^{-4}$ with $80 \mathrm{~K}$ training steps in fine-tuning the parameters of the whole model. In addition, the duration predictor is trained with a fixed learning rate of $10^{-4}$ and $10 \mathrm{~K}$ training steps.

\subsection{Evaluation}

In order to evaluate the performance of our model, we use the text transcripts in test datasets as the input of the model, and obtain the synthetic audios, which are rated together with the ground truth audio (GT) by 50 native English speakers. And then the mean opinion score (MOS) is calculated as the evaluation indicator of the text-to-speech systems. As comparative experiments, the audios generated from mel-spectrum of the ground truth audio (GT Mel), Tacotron2 [4], Transformer TTS [9] and FastSpeech [14] are also rated together. The vocoder is implemented using the WaveGlow [12] in these experiments. The results are shown in Table 1, where the time cost for generating one speech is also illustrated. It can be seen that our proposed model gets slightly improved performance than Tacotron 2 and Transformer TTS, and a significant speed boost is acquired in inference. Specifically, it takes only 0.18 seconds to synthesize approximately $10 \mathrm{sec}-$ onds of speech in our model, of which about 0.06 seconds for AlignTTS and about 0.12 seconds for WaveGlow.
Table 1. The comparison of MOS and time cost.

\begin{tabular}{lcc}
\hline Method & MOS & Time Cost (s) \\
\hline GT & $4.53 \pm 0.05$ & - \\
GT Mel & $4.28 \pm 0.08$ & $0.12 \pm 0.02$ \\
Tacotron2 & $3.96 \pm 0.13$ & $4.58 \pm 2.87$ \\
Transformer TTS & $4.02 \pm 0.15$ & $3.26 \pm 1.93$ \\
FastSpeech & $3.88 \pm 0.11$ & $0.16 \pm 0.04$ \\
\hline AlignTTS & $\mathbf{4 . 0 5} \pm \mathbf{0 . 1 2}$ & $0.18 \pm 0.04$ \\
\hline
\end{tabular}

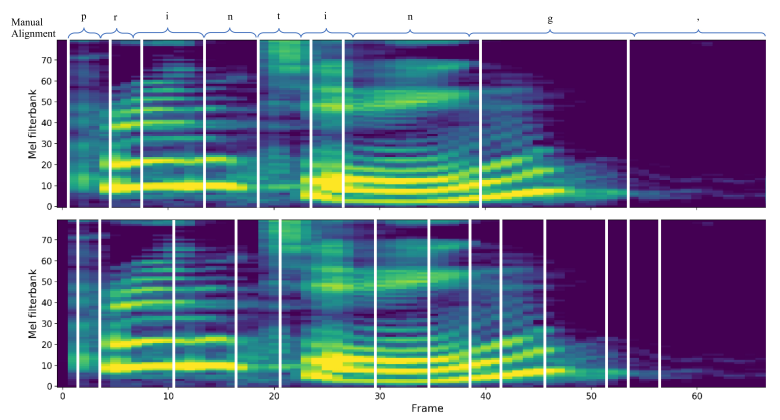

Fig. 3. Comparison of Alignments. The mel-spectrum is split by the alignment of AlignTTS (above) and FastSpeech (below), respectively. The corresponding text is " printing, ...”.

\subsection{Comparison of Alignment}

We design the alignment loss to learn the alignment, while FastSpeech extract it from Transformer TTS model. Comparison of the alignment between our model and FastSpeech is shown in Figure 3. We can find that the alignment from our model is more precise in aligning characters and melspectrum, which effectively guides the training and makes model more excellent and robust than other TTS systems.

\section{CONCLUSION}

In this work, we propose AlignTTS to predict the melspectrum in parallel, and the alignment loss to make model capable of learning the alignment between text and melspectrum. Experiments on the LJSpeech dataset show that the more precise alignment is obtained, which allows AlignTTS to better learn the transformation from characters to melspectrum, and the state-of-the-art performance is achieved.

\section{ACKNOWLEDGEMENTS}

This paper is supported by National Key Research and Development Program of China under grant No. 2018YFB1003500, No. 2018YFB0204400 and No. 2017YFB1401202. Corresponding author is Jianzong Wang from Ping An Technology 
(Shenzhen) Co., Ltd.

\section{REFERENCES}

[1] Jose Sotelo, Soroush Mehri, Kundan Kumar, Joao Felipe Santos, Kyle Kastner, Aaron Courville, and Yoshua Bengio, "Char2wav: End-to-end speech synthesis," in ICLR Workshop, 2017.

[2] Yaniv Taigman, Lior Wolf, Adam Polyak, and Eliya Nachmani, "Voiceloop: Voice fitting and synthesis via a phonological loop," in International Conference on Learning Representations (ICLR), 2018.

[3] Yuxuan Wang, RJ Skerry-Ryan, Daisy Stanton, Yonghui $\mathrm{Wu}$, Ron J Weiss, Navdeep Jaitly, Zongheng Yang, Ying Xiao, Zhifeng Chen, Samy Bengio, et al., "Tacotron: Towards end-to-end speech synthesis," in Annual Conference of the International Speech Communication Association (Interspeech), 2017.

[4] Jonathan Shen, Ruoming Pang, Ron J Weiss, Mike Schuster, Navdeep Jaitly, Zongheng Yang, Zhifeng Chen, Yu Zhang, Yuxuan Wang, Rj Skerrv-Ryan, et al., "Natural tts synthesis by conditioning wavenet on mel spectrogram predictions," in International Conference on Acoustics, Speech and Signal Processing (ICASSP), 2018.

[5] Sercan Ö Arik, Mike Chrzanowski, Adam Coates, Gregory Diamos, Andrew Gibiansky, Yongguo Kang, Xian $\mathrm{Li}$, John Miller, Andrew Ng, Jonathan Raiman, et al., "Deep voice: Real-time neural text-to-speech," in International Conference on Machine Learning (ICML), 2017.

[6] Sercan Ö Arik, Gregory Diamos, Andrew Gibiansky, Gregory Diamos, John Miller, Kainan Peng, Wei Ping, Jonathan Raiman, and Yanqi Zhou, "Deep voice 2: Multi-speaker neural text-to-speech," in Advances in Neural Information Processing Systems (NIPS), 2017.

[7] Wei Ping, Kainan Peng, Andrew Gibiansky, Sercan O Arik, Ajay Kannan, Sharan Narang, Jonathan Raiman, and John Miller, "Deep voice 3: 2000-speaker neural text-to-speech," in International Conference on Learning Representations (ICLR), 2018.

[8] Wei Ping, Kainan Peng, and Jitong Chen, "Clarinet: Parallel wave generation in end-to-end text-to-speech," in International Conference on Learning Representations (ICLR), 2018.

[9] Naihan Li, Shujie Liu, Yanqing Liu, Sheng Zhao, Ming Liu, and M Zhou, "Neural speech synthesis with transformer network," in AAAI Conference on Artificial Intelligence (AAAI), 2019.
[10] Aron van den Oord, Sander Dieleman, Heiga Zen, Karen Simonyan, Oriol Vinyals, Alex Graves, Nal Kalchbrenner, Andrew Senior, and Koray Kavukcuoglu, "Wavenet: A generative model for raw audio," in 9th ISCA Speech Synthesis Workshop, 2016.

[11] Nal Kalchbrenner, Erich Elsen, Karen Simonyan, Seb Noury, Norman Casagrande, Edward Lockhart, Florian Stimberg, Aaron van den Oord, Sander Dieleman, and Koray Kavukcuoglu, "Efficient neural audio synthesis," in International Conference on Machine Learning (ICML), 2018.

[12] Ryan Prenger, Rafael Valle, and Bryan Catanzaro, "Waveglow: A flow-based generative network for speech synthesis," in International Conference on Acoustics, Speech and Signal Processing (ICASSP), 2019.

[13] Kainan Peng, Wei Ping, Zhao Song, and Kexin Zhao, "Parallel neural text-to-speech," arXiv preprint arXiv:1905.08459, 2019.

[14] Yi Ren, Yangjun Ruan, Xu Tan, Tao Qin, Sheng Zhao, Zhou Zhao, and Tie-Yan Liu, "Fastspeech: Fast, robust and controllable text to speech," in Advances in neural information processing systems (NIPS), 2019.

[15] Keiichi Tokuda, Yoshihiko Nankaku, Tomoki Toda, Heiga Zen, Junichi Yamagishi, and Keiichiro Oura, "Speech synthesis based on hidden markov models," Proceedings of the IEEE, vol. 101, no. 5, pp. 12341252, 2013.

[16] Paul Taylor, Text-to-speech synthesis, Cambridge university press, 2009.

[17] Leonard E Baum, Ted Petrie, George Soules, and Norman Weiss, "A maximization technique occurring in the statistical analysis of probabilistic functions of markov chains," The annals of mathematical statistics, vol. 41, no. 1, pp. 164-171, 1970.

[18] Ashish Vaswani, Noam Shazeer, Niki Parmar, Jakob Uszkoreit, Llion Jones, Aidan N Gomez, Łukasz Kaiser, and Illia Polosukhin, "Attention is all you need," in Advances in neural information processing systems (NIPS), 2017.

[19] Christopher M Bishop, Mixture density networks, Aston University, 1994.

[20] Heiga Zen and Andrew Senior, "Deep mixture density networks for acoustic modeling in statistical parametric speech synthesis," in International conference on acoustics, speech and signal processing (ICASSP), 2014.

[21] Keith Ito, "The lj speech dataset," https://keithito.com/ LJ-Speech-Dataset/, 2017. 